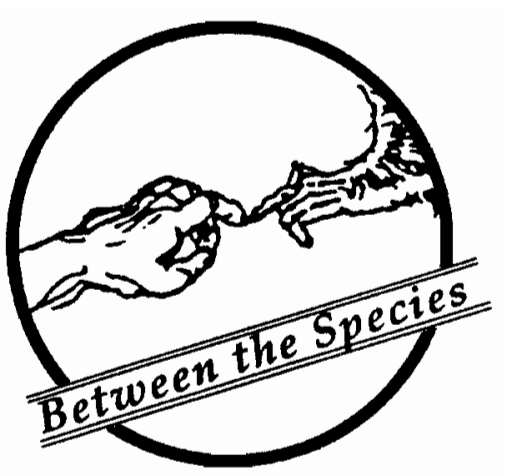

\title{
NATURE VS. NURTURE REVISITED
}

\author{
E. R. Klein \\ Rollins College
}

\section{A review of John Tyler Bonner's The Evolution of Culfure in Animals (Princeton: Princeton University Press, 1980)}

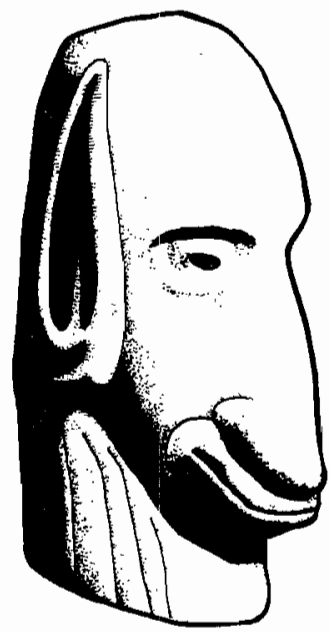

\section{Nature Vs. Nurture Revisited}

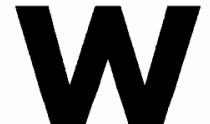

hen biologists turn their attention to the relationships between mankind and animals, they see in mankind the quantitative extensions of qualities present in other species. Social scientists, on the other hand, see the uniqueness of mankind." 2 Bonner is a biologist. And his book, The Evolution of Culture in Animals (ECA), is an attempt to demonstrate that culture, in mankind, is simply a quantitative extension of culture in animals.

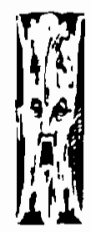

REVIEW

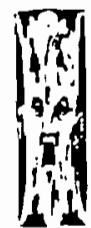




\section{$\underline{\text { Summary }}$}

I n Chapter One, "Philosophy and Less Grand Matters," Bonner states both the purpose and principal conclusion of his book. The purpose is to "trace the origins of the human cultural capacity back into early biological evolution" (3, my emphasis). And, the principal conclusion is that "even though culture itself does not involve genetic inheritance or, therefore, Darwinian evolution by natural selection, the ability of any animal to have culture is a direct product of such an evolutionary mechanism" (3).

In addition to the above, Bonner discusses the holism vs. reductionism debate in science. He sees the debate in its most pedestrian light: Reductionists in science attempt to understand "emergent" 3 properties strictly in terms of their constituent parts. The holist, on the other hand, does not limit herself in this way.

Bonner claims that "both [approaches to science] are important ... [that is] one cannot do without the other" (9). It is suggested that ECA offers an account of culture which will be acceptable to both parties in the debate.

Finally, Bonner defines culture: "By Culture I mean the transfer of information by behavioral means, most particularly by the process of teaching and learning" (10). This definition, according to Bonner, emphasizes the method of transfer, not the kind of information that is transferred. And he calls the transfer of information, by way of this (behavioral) method, cultural evolution.

In Chapter Two, "Cultural and Genetical Evolution," Bonner restates the purpose of his book in a slightly different way. He claims that the purpose of this book is to give an answer to the following question: Why do we, humans, have culture at all?

Bonner claims that there are two possible approaches one might take in attempting to answer this question. The first approach is to examine those features of culture that are selectively advantageous. The second possible approach is to examine the early evolutionary origins of culture. Bonner chooses to follow both paths, and the bulk of ECA is an interesting account of this journey.
Before beginning to trace his steps, though, Bonner offers the reader an account of the basic similarities and, more importantly, the basic distinctions between cultural and genetical evolution. While both forms of evolution transmit information, genetical evolution transmits information via the gene, ${ }^{4}$ while cultural evolution transmits information via beliefs, ideas, customs, etc., what Bonner calls a meme. ${ }^{5}$

This nominal distinction does not, according to Bonner, reflect a difference in kind but simply a difference in degree, and it is suggested that cultural evolution, being a higher degree of evolution, is better. Genetical evolution is considered to be a lower degree of evolution because of specific limitations. Genes limit an individual both in the speed in which it can transmit information from itself to another individual and in the amount of individuals it can transfer information to in its lifetime. After all, the transfer of information via genes requires reproduction. Cultural evolution, on the other hand, is not so restricted - a meme does not limit the transfer of information in either of these two ways. Memes can be transmitted by an individual to a very large number of individuals and this can occur very quickly.

In Chapter Three, "The Brain and the Genome," Bonner begins his discussion of the early evolution of culture by tracing the steps of the progression of brains. The brain, he correctly claims, is a product of genes. Therefore, given that animals "higher on the evolutionary scale" have bigger brains than those animals with a lower standing, a bigger brain (most probably) is a product of natural selection. That is, "during the course of genetical evolution, selective pressure for the machinery must have produced the fast information processor of the nervous system. This ultimately led to a progressive centralization of the nervous system (still by natural selection of genes) leading to larger brains and finally to those capable of inventing culture" (33, my emphasis).

Therefore, Bonner concludes, brain size ${ }^{6}$ is a direct outcome of evolution. After all, "this selective advantage of larger brains is evident in the evolution of vertebrates. There is a direct inverse correlation with the time of appearance 
of a group in earth history and the size of its brain. At one end of the spectrum fish have small brains, while on the other end mammals have the largest" (45).

Furthermore, according to Bonner, the increase in the size of the brain suggests "a trend toward increase in ability to learn, toward increase in flexibility of response" (45). And such flexibility is a necessary condition for the development of culture.

It is suggested, therefore, that if the increase in flexibility of response can be traced from humans back into the most primitive species, then two claims become plausible. The first claim is that only animals have the capacity for culture, the second, that culture (in animals) evolved.

In Chapter Four, "The Early Origins of Cultural Evolution," Bonner offers an interesting and informative account of culture in animals. Given that animals, even animals as primitive as bacteria, can, unlike plants, move, and are therefore capable of behavior, Bonner claims that they are our cultural ancestors.

Bonner attempts to make this claim plausible in the following way. He restates the distinction between cultural and genetical evolution. The distinction, he claims, "resides in a quick, flexible response for cultural evolution and a slow, ponderous response for genetical evolution..." (56). Then, given the fact that the quickest response that an animal can take to its environment is a behavioral response, it is suggested that merely the ability to behave is a necessary condition for cultural evolution. Plants cannot evolve culturally; only animals can. Bacteria, being motile, qualify as animals. Therefore, bacteria qualify as our cultural ancestors.

But the kind of culture of which bacteria are capable is certainly a far cry from the kind of culture of which humans are capable. Bonner, of course, recognizes this. Therefore, Chapter Five, "The Evolution of Animal Societies," attempts to lend support to the claim that the characteristic of being social is a recessary condition for a more sophisticated notion of culture. But, certain properties indicative of human culture (e.g., the ability to communicate) is apparent in many animal societies (e.g., ants, wolves and humans), though at varying degrees. Thus, it is plausible to claim that this characteristic has adaptive advantage. And if this is the case, then it is plausible to conclude that this social characteristic (a necessary condition for culture) evolved.

Bonner begins this chapter by defining an animal society as a "cohesive group of intercommunicating individuals of the same species" (76). And it is this communicating characteristic, shared by all of the individuals in the group, which is the essence of the society. Therefore, given that culture is defined as "the transfer of information by behavioral means, most particularly by the process of teaching and learning..." (76), along with the above definition of a society, then if it can be demonstrated that the characteristic of being social is a product of evolution, then it would be plausible that culture, which is "simply not possible without communication" (113), is also a product of evolution.

Given that Bonner has demonstrated that social behavior (e.g., cooperative behavior) is (most likely) a consequence of natural selection, Bonner suggests that he has offered evidence for at least the plausibility of the thesis that the ability for culture itself is a consequence of natural selection. ${ }^{7}$

In Chapter Six, "Learning and Teaching," Bonner discusses that aspect of communication behavior that he claims is the hallmark of a sophisticated) culture, namely, teaching and learning. Bonner traces the evolution of both.

The evolution of learning (from the most primitive "learners" to the most advanced) is the following: (1) Organisms that can respond to the environment, but only in a "fixed" way, e.g., "the shooting out of the proboscis of a fly in the presence of sugar water" (134). (2) Organisms which respond to the environment in a way that is limited but not fixed, e.g., "a fly moving toward or away from light" (135). (3) Organisms which can demonstrate a "slight flexibility in the response by being continuous over a range of related but quantitatively varied stimulus ... a good example would be the honey bee's response to signals indicating different degrees of distance or direction" (135). (4) Organisms which can only respond (correctly) to the environment by learning the correct response "rather than [by giving] an automatic, innate fixed 
response" (135). An example of this kind of response is apparent in the development of different (song) "dialects" within the same species of song-bird.

The evolution of teaching (from the most primitive "teacher" to the most advanced) is the following: (1) Organisms which can produce only a single signal, directed toward one individual. (2) Organisms which can produce more than one signal. (3) Organisms which can produce a "great proliferation of these signals that become increasingly loaded with information" (135). (4) Organisms which can put their signals into artifacts such as writing.

In Chapter Seven, "The Evolution of Flexible Response," Bonner attempts to demonstrate that "culture and its transmission is the ultimate in flexible behavior" (137), in order to help defend his original thesis, that culture is (indirectly) a product of natural selection. Toward this end, Bonner demonstrates that flexible response itself, evident in all animals, particularly mammals, and most particularly in humans, evolved.

It is interesting to note that Bonner concentrates his discussion on those flexible responses surrounding mating and offspring-rearing behaviors. In a discussion of the numerous "strategies" which animals in fact use to achieve the desired result of a new generation, Bonner stresses the point that within the same species there often are alternative (and even contradictory, e.g., monogamy and polygamy) strategies for successful mating and rearing. He calls these (successful) alternative strategies "evolutionary stable strategies" (160).

Whether these strategies are determined by gene or meme, at this point, is irrelevant. The conclusion which Bonner suggests is that, at least from the point of view of survival value, ${ }^{8}$ any (actual or possible) strategy, if evolutionarily stable, is as good as any other.

This, of course, holds for human evolutionary stable strategies as well. Therefore, for example, as long as children are born ${ }^{9}$ and raised, it is irrelevant whether they are born or raised by men or women or some combination of the two.

The last chapter, Chapter Eight, "The Evolution of Culture," traces the evolutionary progression of culture (i.e., its degree of complexity) by tracing the evolutionary progression of animals, specifically the development of their brains. (Also, a conclusion to the book is given at the end of this chapter.)

\section{Instead of asking whether a particular behavior is a product of genes or environ- ment, one can ask a similar question about human behavior as a whole.... Given that there is such an "enormous increase" in the amount and complexity of culture (between humans and other animals), does this difference in degree justify one in believing that there is a difference in kind?}

In this chapter Bonner concentrates the discussion of evolutionary progression on that portion of the "evolutionary chain" that is nonhuman. Bonner offers many examples of culture in nonhumans in order to establish that culture, like the animals in which it is present, has progressed. He offers examples of culture in the very primitive culture in bacteria, progressing through the less primitive culture in insects and reptiles, up to the even less primitive culture in 
primates, to the advanced culture of Homo sapiens, finally culminating in our modern (literate) culture.

Bonner's main point here is that the parallel between the evolution of animals and the evolution of culture suggests that if the former is progressive, 10 the latter must be so as well. Therefore, culture is (nost likely) a product of natural selection.

Parenthetically, Bonner recognizes that although the progression of culture in bacterial "societies" to the culture in, for example, primate societies is impressive, the progression of culture in primate societies to the culture in human societies is much more so. "In an evolutionary progression, if one passes from primates to man, the amount and complexity of the culture increases enormously" (185).

Bonner concludes the book with the following disclaimer: "In the case of man ... it is especially difficult to demonstrate what components of behavior have a direct genetic basis" (195). But, it is suggested that this difficulty concerning a particular behavior does not affect his claims concerning behavior in general. Consequently, he claims that instead of asking whether a given behavior $\mathrm{X}$ has a direct genetic basis, the most "rewarding" question which we could ask about culture is, "Why did this mode of transmission [by memes] arise in the first place during the long course of evolution" (196)?

Bonner's answer to this question is "that culture as a process is by itself of enormous adaptive value" (196). Therefore, an animal with the ability for culture (the ability to adapt to situations with enormous flexibility), i.e., a big brain, would have selective advantage. Therefore, "culture lies in the genetical evolution of animals and man" (198).

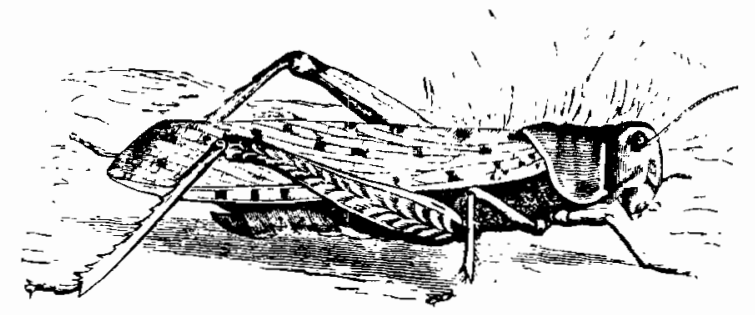

\section{The Argument}

D onner's argument in support of the above claim is bifurcate. The first part of the argument is used to establish the fact that bigger brains (brains that are large relative to the animals' body weight) are, from the point of view of evolution, better brains. The argument is the following: (1) [P] resumably all the major physical features of our brain are genetically determined" (25). (2) Brain size is a major physical feature, and "the selective advantage of larger brains is evident" (45). (3) Therefore, larger brain size is the proper product of progenitive progress.

The second part of the argument attempts to establish the fact that an animal's having a large brain is, at least, a necessary condition for that animal's having the capacity for culture. The argument is as follows: (1) In order to have the capacity for culture the animal must have the capacity to communicate with members of its own species (113, paraphrase). (2) ln order to communicate, animals must be able to transfer certain kinds of information quickly and easily. (3) This ability requires that the animal have a large brain. Therefore, (4) Having a large brain is, at least, a necessary condition for having the ability for culture.

In the light of the above arguments, Bonner's claim that the ability for culture is genetically determined is true but trivial. And it is trivial in two senses. It is logically trivial: If having a large brain is, at least, a necessary condition for having the ability for culture and if larger brain size is a product of genetics, then it simply follows that the ability for culture is a direct product of evolution.

But, more importantly, this claim is conceptually trivial; that is, it is uncontentious. Who would doubt that the ability for culture in animals was a product of natural selection? Or, who would debate the fact that culture is "indirectly" a product of natural selection? After all, what other choice is there, God?

The important point is that anyone who accepts the theory of evolution as the correct explanation for why humans (or any other animals) exist at all believes that all of our 
physical characteristics (and, therefore, the physical characteristic of a big brain) were (at least) not unconducive to our survival. Therefore, given that the ability for culture is ultimately grounded in the physical characteristic of a big brain, it is a trivial claim to state that the ability for culture is (most likely) a product of natural selection. I cannot imagine that anyone (except maybe a "Creationist"), let alone any sociologist, would find this very weak claim to be at all contentious.

The debate between the sociobiologist and the sociologist revolves around the question of whether or not our (human) particular social activities (e.g., monogamy) are a product of "nature" or "nurture." Bonner avoids making a claim, either way, on this point. But he does not avoid the issue altogether. Instead of taking a stand, Bonner offers a reason why he has avoided this issue; namely, regardless of which side of the issue one stands on such a determination is "intractable" (188).

But the debate can still be waged on a more general level. Instead of asking whether a particular behavior is a product of genes or environment, one can ask a similar question about human behavior as a whole. The debate between the sociobiologist and the sociologist would then center around the following kind of question: Given that there is such an "enormous increase"ll in the amount and complexity of culture (between humans and other animals), does this difference in degree justify one in believing that there is a difference in kind? Bonner simply avoids this kind of question.

Furthermore, Bonner even avoids the weaker, heuristic question: Does this difference in degree (whether indicative of a difference in kind or not) justify one in treating the discussion of culture in humans differently, that is, as if there was a difference in kind? It is this latter kind of question which makes for interesting discussion. Bonner (in ECA) has simply avoided the interesting.

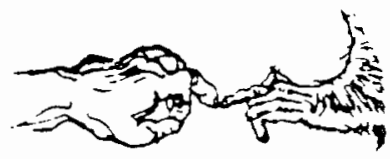

\section{Further Remarks}

. $n$ light of the above I think that it is important to try to understand why (and for whom) Bonner would have written ECA. For Bonner himself recognizes that the thesis that "the capacity for culture undoubtedly has a genetic basis ... [is a thesis with] which everyone agrees ... [for] this could be no more than saying that the genes determine the structure of the brain" (29).

In attempting to offer an answer to the question, 'Why has culture come into being?,' Bonner offers such a weak answer in order to demonstrate that one can stand on neutral ground concerning the debate between the sociobiologist and the sociologist. In so doing he attempts to convince proponents from both sides of the issue to erase the "battle lines" (187) between biology and the social sciences. Bonner believes that "it is important for the biologist, and more particularly the sociobiologist, to realize that his recent flashes of insight that have come, for instance, through the aegis of kin selection, will not solve all the problems of the social sciences, but may shed some bright light on aspects of human social behavior. The social scientist, on the other hand, must face the possibility of some biological information being extraordinarily useful to him, and certainly it should not be rejected for doctrinaire reasons" (187).

But although Bonner may be correct that a spirit of comradery between the scientists involved in these two camps is necessary, it does not follow from this that there should be a breakdown of all distinctions between the two disciplines. After all, the questions (answers) of one discipline may not be relevant ${ }^{12}$ to the questions (and answers) of the other. At least, such relevance has not been demonstrated by Bonner.

Furthermore, Bonner simply assumes that a proper answer to the question, "Is culture, as a means of non-genetic transmission of information, adaptive" (188)?, requires an interdisciplinary approach. And this point, though not debated in ECA, is certainly debatable. ${ }^{13}$ 
Therefore, although Bonner may be right that the only intolerable position concerning the nature vs. nurture issue is that of either extreme - "that all or no cultural phenomena have a direct genetic involvement" (32) - this point has not been demonstrated.

ECA offers the reader an interesting journey through the development of culture in animals. But although there is much here that may be of interest to the biologist, there is little here of philosophical or political value. Most importantly, there is nothing in ECA which the sociologist need worry about. Even if everything that Bonner suggests is true, none of these facts speaks to the real question: Is human culture a product of nature or nurture?

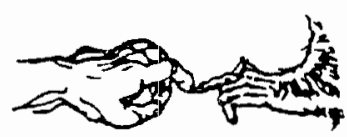

$\underline{\text { Notes }}$

${ }^{1}$ All textual page references are to this work.

2 R. Haven Wiley, "The Capacity for Culture," Science, July 1980.

${ }^{3}$ Bonner claims to use the term "emergent" in the "straightforward" sense. That is, an emergent property is any new property, either structural or behavioral, which did not exist "at a lower level of complexity." (p. 186)

4 This is the standard account of genes. Information is encoded in the molecular structure of cells (DNA) and is then passed on from one generation to another.

${ }^{5}$ R. Dawkins, The Selfish Gene, 1976.

${ }^{6}$ That is, brain size relative to body size.

7 In the last chapter of the book, specifically p. 193, Bonner makes this claim more clear when he states that "the system of communication itself has spawned the possibility of a new method of transmission [of information] bypassing the genes. This means that in order to win in the struggle for reproductive success it might be advantageous to transmit information directly from one individual to another rather than through the genome. Therefore any cooperative or selfish act that is adaptive and that could be achieved by quick signal transmission would be favored over any slower genetic transmission. This bypassing was so successful that the selective process in the genes was no longer for more elaborate genetic signals, but for bigger brains that could transmit a wide variety of rapid, flexible, innovative signals in a behavioral, rather than in a genetic fashion. This step is the cornerstone of the evolution of culture; and there is every reason to believe it occurred as the consequence of natural selection."

"Survival value is the only "value" which Bonner acknowledges.

9 Of course one can imagine an external (artificial) womb or a "natural" womb implanted in a male.

${ }^{10}$ Very few would doubt that humans are the most evolutionarily advanced species.

${ }^{11}$ This great increase in the amount and complexity of culture from primates to man (indicative in man's ability to teach and learn via language) is recognized by Bonner, p. 185. My point (below) is that Bonner does not take a stand as to whether this enormous difference in degree justifies us in believing (or not) that there is a difference in kind.

${ }^{12}$ By relevant I mean that the sociological information must provide good reason for accepting or rejecting a particular sociobiological claim, or vice versa. That is, the findings of sociology (or sociobiology) are only relevant to the findings of sociobiology (or sociology respectively) if they give us good reasons for believing that the claims made by the latter are true.

${ }^{13}$ Actually, I think that the question of whether sociobiology (or sociology) is relevant (in the relevant way to sociology or sociobiology respectively) is a philosophical question! 